\title{
Development and Biomechanical Analysis of a New Anterior Cervical Pedicle Screw Fixation System
}

\section{Maji Sun}

Xuzhou Medical College Affiliated Hospital

\section{Qiuan Wang}

Xuzhou Medical College Affiliated Hospital

Xingchen Zhang

Xuzhou Medical College Affiliated Hospital

\section{Rui Zhao}

Sixth Peoples Hospital of Kunshan

\section{Kaijin Guo}

Nanjing Medical University

Feng Yuan (D 562622911@qq.com )

Xuzhou Medical College Affiliated Hospital

\section{Research article}

Keywords: Anterior cervical spine, Transpedicle, Internal fixation system, Biomechanics

Posted Date: January 21st, 2021

DOl: https://doi.org/10.21203/rs.3.rs-150237/v1

License: (c) (1) This work is licensed under a Creative Commons Attribution 4.0 International License.

Read Full License 
Development and biomechanical analysis of a new anterior cervical pedicle screw fixation system

\section{Author:}

First author: Maji Sun (postgraduate)

Author unit: Nanjing Medical University(China)

mailbox:376333460@qq.com

Co-first author: Qiuan Wang(undergraduate)

Author unit: Xuzhou Medical University Affiliated Hospital(China) mailbox:634001275@qq.com

Third author: Xingchen Zhang(postgraduate)

Author unit: Xuzhou Medical University Affiliated Hospital(China) mailbox:yijiqianli@qq.com

Fourth author: Rui Zhao(undergraduate)

Author unit: The Sixth People's Hospital of Kunshan (China)

mailbox:1391871575@qq.com

Fifth author: Kaijin Guo(doctor)

Author unit: Nanjing Medical University(China) mailbox:301810212309@stu.xzhmu.edu.cn

Corresponding author: Feng Yuan*(doctor)

unit: Xuzhou Medical University Affiliated Hospital(China) mailbox:562622911@qq.com

\section{Funding/Support and Role of Sponsor:}

Jiangsu Science and Technology Department Project ( Social development-Clinical Frontier Technology, NO: BE2016647).

【Abstract】 Objective A new anterior cervical pedicle screw fixation system was developed based on the relevant anatomical structure of the cervical spine, and its biomechanical properties were evaluated on fresh cadaver cervical spine specimens to provide a basis for preliminary clinical application. Methods Three-dimensional parameters of the new nail plate system were obtained from the anatomical data of cervical spine specimens, and the system was produced by 3D printing technology. Fresh adult cadaver cervical spine specimens were used to measure biomechanical stability in the intact state (group A: complete group) and to establish a C5-7 instability model in which fixation with the traditional cervical anterior nail plate system was performed (group B). Anterior cervical spine displacement, strength, stiffness, torsion torque, etc. were measured under the fixed state when 4 screws were used for fixation with the pedicle screw system (group C) and 6 screws were used for the anterior cervical pedicle screw system (group D). Results In terms of the load-displacement relationship, the results showed that the average displacement difference between group D and group C after the test was $25 \%$, that between group D and group B was $30 \%$, that between groups $\mathrm{C}$ and $\mathrm{B}$ was $18 \%$, and the differences were statistically significant $(\mathrm{P}<0.05)$. In terms of the axial stiffness of the cervical spine, that of group D was $20 \%$ higher than that of group $\mathrm{C}$ and $40 \%$ higher than that of group B, that of group C was $20 \%$ higher than that of group B when fixed, and those of group D and $\mathrm{C}$ were both better than that of group $\mathrm{A}$, the blank control group; all the differences were statistically significant $(\mathrm{P}<0.05)$. In terms of torsion mechanical properties of the cervical spine, the experimental results showed that that of group $\mathrm{D}$ was higher than that of group C by $21 \%$ and higher than that of group B by $40 \%$, that of group $\mathrm{C}$ was higher than that of group $\mathrm{B}$ by $30 \%$, and the differences were statistically significant $(\mathrm{P}<0.05)$. The biomechanical tests showed that in terms of load-displacement, axial stiffness and torsional mechanical properties, the anterior cervical pedicle locking and guiding internal fixation system was slightly better than the 
complete method. The 6 nail fixation method was better than the 4 nail fixation method. Conclusion The new anterior cervical pedicle screw fixation system conforms to the anatomy of Chinese individuals and has better mechanical stability than do traditional fixation methods. It is a new, reliable anterior cervical pedicle internal fixation system for clinical practice. List of Abbreviations: ACPS: anterior cervical pedicle screw.

【Key words】 Anterior cervical spine; Transpedicle; Internal fixation system; Biomechanics

\section{Introduction}

Anterior cervical discectomy with bone graft fusion is a common surgical procedure performed for the treatment of cervical spondylosis [4,5]. Because of its high bone graft fusion rate and high stability, it is widely used in clinical practice. However, when surgery for two or more segments is performed, because the bone graft needs to span a large area, poor stability between the internal fixation and the bone graft, bone graft fusion failure and false joints occur. The probability of such complications occurring is very high [6-9]. To improve the stability of anterior cervical discectomy and bone grafting and fusion, Koller [10] and other scholars proposed the anterior cervical pedicle screw (ACPS) technique in 2008. The anterior and posterior approach was performed through anterior surgery and yielded good joint internal fixation stability [1,2]. Another group of scholars, Zhao et al. [11], began to use ACPS in clinical practice in China and observed good results. However, the current clinically applicable anterior cervical nail plate system is suboptimal, and it is difficult to widely promote this technology.

In this experiment, a new type of cervical spine anterior transpedicular locking guided internal fixation system was designed based on the results of a Chinese cervical spine anatomy study [3], which showed that the spine can be fixed with anterior cross pedicle screws or anterior unilateral pedicle screws and vertebral screw fixation. This experiment used fresh human cervical spine specimens to study the biomechanical properties of different versions of the new anterior cervical pedicle internal fixation system, and the results provide a basis for preliminary clinical applications.

\section{Materials and methods}

1.1 Design 1. The ACPS fixation system was designed based on Chinese anatomical characteristics.

2. Biomechanical analysis was performed on fresh corpse specimens.

1.2 Time and place The experiment was performed from 2018.12-2019.07 at Xuzhou Medical University Orthopaedic Laboratory.

\subsection{Materials}

\subsubsection{Development of a new anterior cervical pedicle locking guided internal fixation system}

Development method Based on the results of a Chinese cervical spine anatomy study [3], a new type of anterior cervical pedicle locking guide internal fixation system was designed, and the system was made using 3D printing technology (Figure 1). In the experiment, a three-dimensional model of the human cervical spine was designed using three-dimensional software and the average anatomy of the cervical spine in Chinese individuals, and a new type of anterior cervical pedicle fixation titanium plate screw was simulated to obtain the locking guide sleeve angle. 

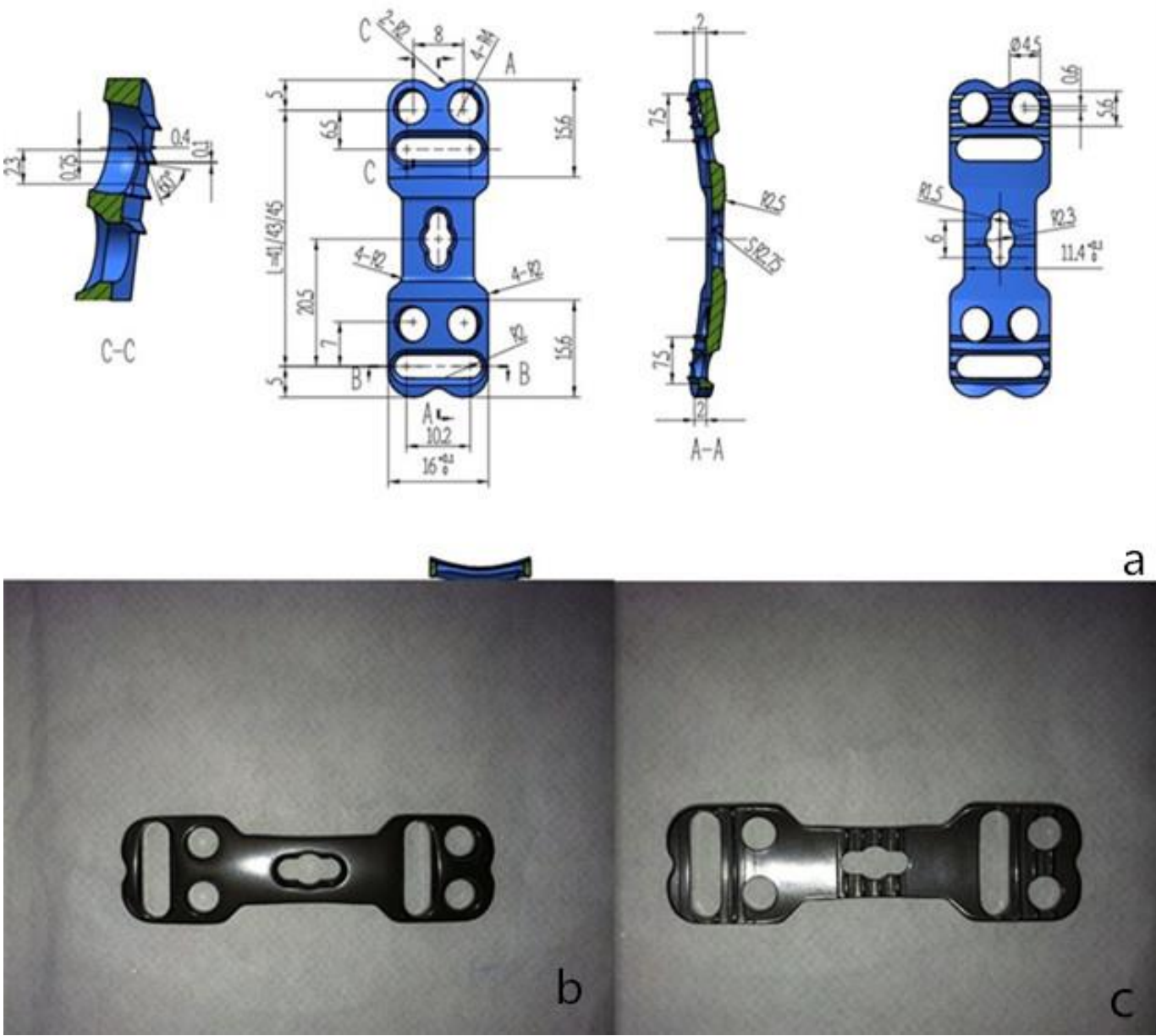

Legend: A. Drawing of the titanium plate design B. Front view of the titanium plate C. Back view of the titanium plate Figure 1 New type of anterior cervical titanium plate

According to the cervical spine anatomy, the width of the two ends of the titanium plate is 16 $\mathrm{mm}$, the middle width is $12 \mathrm{~mm}$, the anterior diameter of the upper screw hole is $12 \mathrm{~mm}$, the lateral diameter of the contact vertebral body (posterior diameter) is $11.5 \mathrm{~mm}$ ), and the lower screw hole spans 2 horizontally distributed circular vertebrae. The diameter of the nail hole is $4.5 \mathrm{~mm}$, the distance between the centres of the two screw holes is $8 \mathrm{~mm}$, and the distance from the centre of the upper screw hole is $7.9 \mathrm{~mm}$, and the two screw holes at the lower end are similar to spherical grooves. The front diameter of the screw hole is $4.5 \mathrm{~mm}$. The back diameter is $3.5 \mathrm{~mm}$, the sidewall thickness of the back diameter is $0.5 \mathrm{~mm}$, the diameter of the fixing screw is $3.5 \mathrm{~mm}$, the thread pitch is 2.45 $\mathrm{mm}$, the thread core diameter is $2.5 \mathrm{~mm}$, the lower end is a single cortex screw, the screw diameter is $3.5 \mathrm{~mm}$, the thread pitch is $2.45 \mathrm{~mm}$, the thread core diameter is $2.5 \mathrm{~mm}$, the thickness of the titanium plate is $3 \mathrm{~mm}$, the arc of the sagittal plane is $9.5^{\circ}$, and the radius is $150 \mathrm{~mm}$. The radius of the coronal surface is $41 \mathrm{~mm}$, and the lower edge of the titanium plate is $155^{\circ}$. In addition, the discontinuous transverse convex design is added to the back of the titanium plate. The upper nail hole diameter is $12 \mathrm{~mm}$, the horizontal centre line is $6.5 \mathrm{~mm}$ from the lower nail hole horizontal centre line, the screw length ranges from $10-16 \mathrm{~mm}$, and the length of the titanium plate ranges from $28-68 \mathrm{~mm}$. 


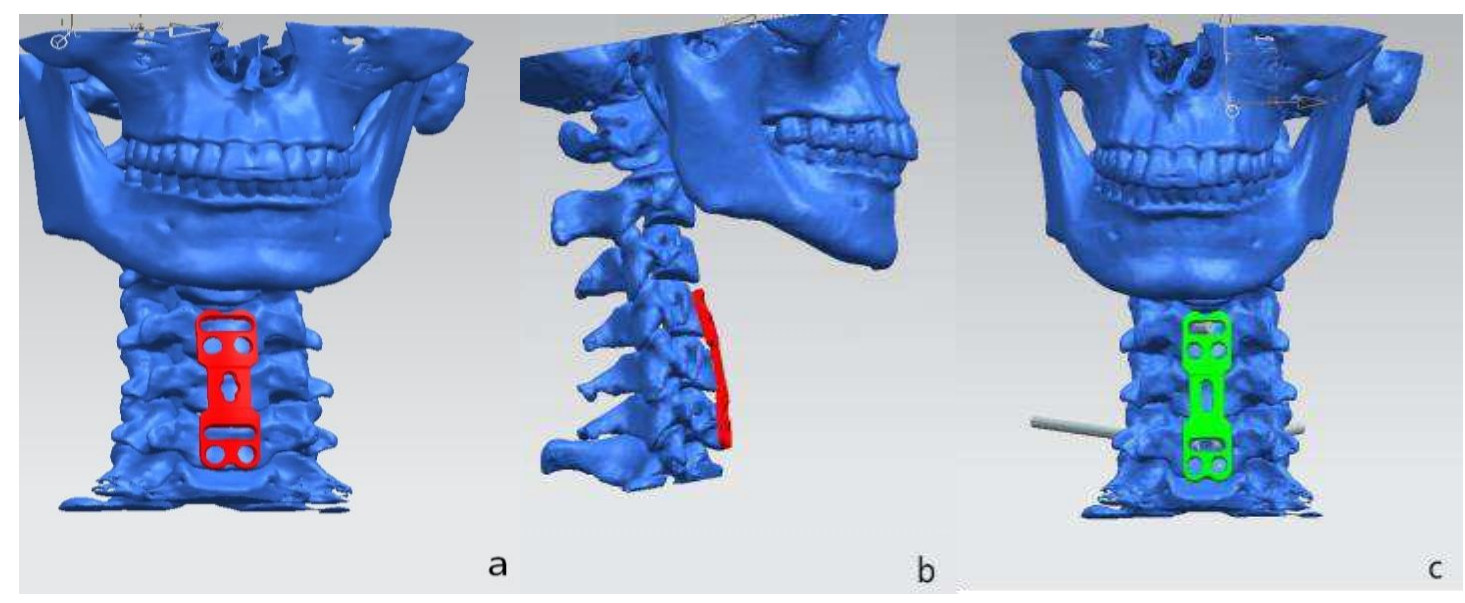

Legend: A Three-dimensional cervical spine simulation showing the placement of the plate; $\mathrm{B}$ Three-dimensional cervical simulation showing the lateral placement of the plate; $\mathrm{C}$ Threedimensional simulation of the locking guide sleeve.

Figure 2: Three-dimensional schematic diagram of the new cervical spine anterior titanium plate fixation method

Structural features The design of the titanium plate conforms to the anatomical characteristics of the Chinese population. The screw holes at both ends are horizontally elliptical, which favourably allows a $47^{\circ}-48^{\circ}$ camber when the pedicle screw is placed, and the best angle of the pedicle screw locking sleeve can be measured preoperatively in three dimensions. During the operation, two pedicle screws can be inserted through the guide locking sleeve, or one pedicle screw can be inserted separately. The side wall thickness of the back diameter of the lower screw hole is $0.5 \mathrm{~mm}$, and the screw thread space is $1.75 \mathrm{~mm}$; therefore, when the two parts are engaged with each other, the long axis of the screw and the plane of the titanium plate form an angle of $15^{\circ}-20^{\circ}$ in all directions. The angle of intersection ensures that the fixation screws at both ends have a head-to-tail angle of $15^{\circ}$ during the operation, and it also allows the screws to have a certain angle of cohesion or a certain angle of abduction, which not only conforms to the "tension band principle" of internal fixation but also makes titanium plate screws difficult to loosen or remove. Three screws are used to fix the cone, which effectively enhances the stability of the fixed segment and the pull-out strength of the titanium plate. The back design of the titanium plate has a discontinuous transverse convex design to make the bone-titanium plate interface rough. This design not only helps prevent slippage but also increases the overall stability of the cervical spine after fusion of the titanium plate and helps prevent bone deficiency in front of the vertebral body and blood necrosis.

1.3.2 Cervical spine specimens Six fresh cadaveric cervical spine (C2-T1) specimens (provided by the Anatomy Department of Xuzhou Medical University) were used, and specimens determined to be noncompliant through lateral X-ray photography and gross anatomy examinations were excluded. There were 2 males and 4 females, aged 38 to 57 years old, with an average of 45.5 years old; the average bone density was 0.687 , all the muscles of the specimens were removed, and all ligaments and small joint capsules of each specimen were retained. In the preparation phase of the experiment, the specimens were sealed in plastic bags and stored in a refrigerator at $-80{ }^{\circ} \mathrm{C}$. Before the experiment, the specimens were removed from the refrigerator and thawed gradually. When the specimen was preserved, it was kept in a natural neutral position. Studies have shown that preservation according to the above method does not change the mechanical properties of the cervical spine $[12,13]$. 
1.3.3 Instruments An X-ray fluoroscopy machine (from the imaging department of the Affiliated Hospital of Xuzhou Medical College), a CTM8010 microcomputer-controlled electronic universal material testing machine (Shanghai Jian Qiang Instrument Manufacturing Co., Ltd.), and a new type of anterior cervical titanium plate were used.

\subsection{Methods}

1.4.1 Experimental grouping In an environment with a room temperature of $20^{\circ} \mathrm{C}$ and a relative humidity of $60 \%$, the 6 processed specimens underwent tests of displacement, strength, and stiffness in 4 states in the following order (Figures 3, 4). Group A was considered the complete group. Group B was the traditional anterior cervical nail plate system fixation group (Watson Medical Equipment Co., Ltd.) (each of the upper and lower 2 fixed vertebral bodies had 2 vertebral screws). Group C was the new type of fixation group with the cervical spine anterior transpedicular internal fixation nail plate system with 4 screws (Watson Medical Instruments Co., Ltd.) (each of the upper and lower 2 fixed vertebrae had 2 pedicle screws). Group D was the fixation group with the new type of cervical spine anterior transpedicular internal fixation nail plate system with 6 screws (Watson Medical Instruments Co., Ltd.) (the upper and lower two fixed vertebral bodies had 1 pedicle screw and 2 vertebral screws).

1.4.2 Biomechanical analysis of the new anterior cervical pedicle locking guide internal fixation system Six specimens with a complete C2-T1 structure underwent measurements of displacement, strength, stiffness, torsion moment, etc. before the complete state (group A). Then, the C6-7 unstable model was made; that is, a $\mathrm{C} 6$ vertebral body subtotal incision was performed anteriorly to cut the C6-7 inter-ligament structure, and the C6-7 supraspinous ligament, interspinous ligament, ligamentum flavum, and joint capsule were incised bilaterally, and the posterior half fibrous ring of the C6-7 intervertebral disc was incised. Displacement, strength, stiffness, torsion torque and other parameters were measured in the fixed state in groups B, C, and D successively to determine the biomechanical performance indexes of the cervical spine under various working conditions.

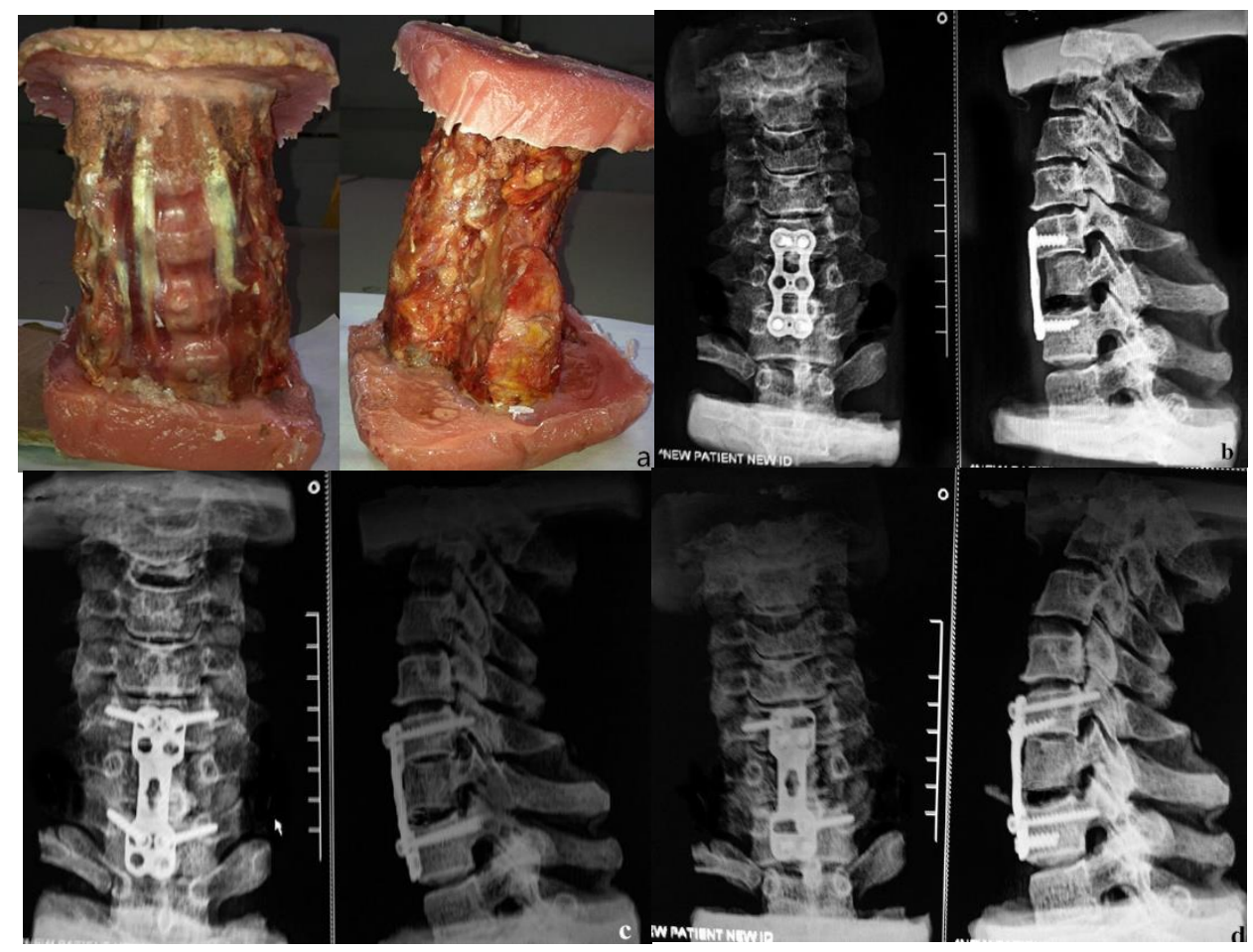


Legend: A. Group A blank control condition; B. Group B fixation method; C. Group C fixation method; D. Group D fixation method

Figure 3: Postoperative $\mathrm{X}$-ray images of each group in the positive and lateral positions

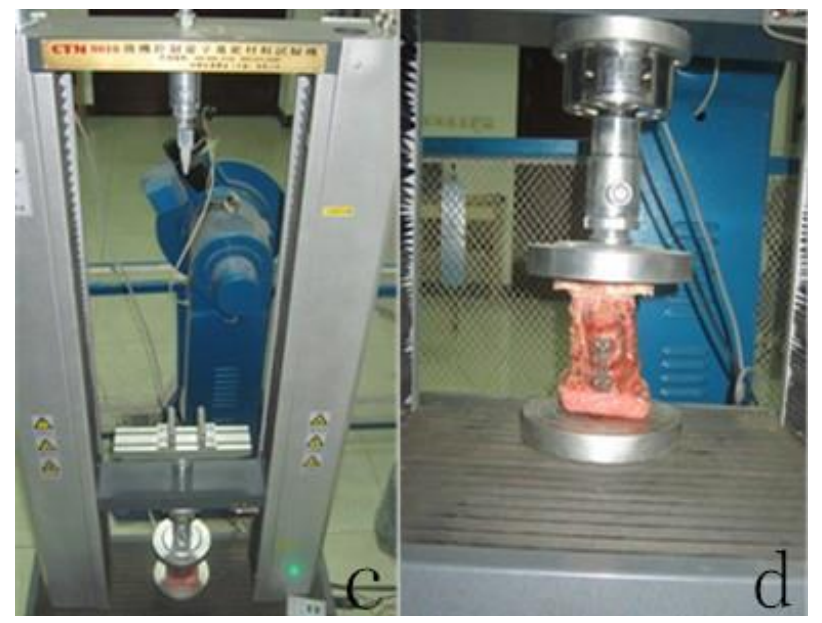

Figure 4 Biomechanical test conducted on a specimen

\subsection{Main indicators observed}

Regarding displacement and torsion mechanics, according to the internal fixation method, the torsion force (Mn) was measured by collecting data during central compression, flexion, extension, and flexion loading.

Regarding the pull-out force, 3 specimens in each group were placed on the CTM-8010 experimental machine, and the titanium plate screw system was pulled out.

For the fatigue test: Sinusoidal loading was induced by the PYW-20 microcomputer to perform a high frequency fatigue test; the number of times the specimen was loaded and the breaking point were recorded; if there is no break, the looseness of the titanium plate and screws was observed;

1.6 Statistical analysis SPSS 16.0 statistical software was used for analysis, and the data are expressed as the mean \pm standard deviation $(\mathrm{x}(-) \pm \mathrm{s})$. The two groups were compared by the $\mathrm{t}$ test and Fisher's exact likelihood method. The level of significance for hypothesis testing was $\alpha=0.05$, and $P<0.05$ was considered statistically significant.

\section{Results}

\subsection{Load-displacement relationship}

The 4 groups were tested under four stresses, axial compression (AC), flexion (Flex), extension (Ext), and lateral flexion (LB), so that the experimental specimens underwent longitudinal displacement and deformation under the abovementioned load (Table 1). The average difference in displacement between group D and group C was $25 \%$, that between group D and group B was $30 \%$, that between group C and group B was $18 \%$, and the difference across groups was statistically significant $(\mathrm{P}<0.05)$.

Table 1 The longitudinal displacement results of the C5-C7 segment under 4 load conditions under $150 \mathrm{~N}$ load $(\mathrm{x}(-) \pm \mathrm{s}, \mathrm{n}=6, \mathrm{~mm})$

\begin{tabular}{lllll}
\hline Exercise type & Group A & Group B & Group C & Group D \\
\hline
\end{tabular}




\begin{tabular}{lllll}
\hline A.C & $3.61 \pm 0.73^{*, \dagger}$ & $2.10 \pm 0.26^{*, \dagger}$ & $1.87 \pm 0.30^{*}$ & $1.14 \pm 0.09^{*}$ \\
Flex & $4.34 \pm 0.46^{*}$ & $3.44 \pm 0.29^{*}$ & $2.87 \pm 0.48^{*}$ & $2.07 \pm 0.28^{*}$ \\
Ext & $3.53 \pm 0.80^{*, \dagger}$ & $2.83 \pm 1.02^{*, \dagger}$ & $2.42 \pm 0.25^{*}$ & $1.80 \pm 0.28^{*}$ \\
LB & $4.42 \pm 0.46^{*}$ & $3.23 \pm 0.36^{*}$ & $2.53 \pm 0.38^{*}$ & $1.73 \pm 0.23^{*}$ \\
\hline
\end{tabular}

The relative differences between the data marked with * are statistically significant $(\mathrm{P}<0.05)$, the differences between the data marked with $*$ and those marked with $*, \dagger$ are statistically significant $(\mathrm{P}<0.05)$, and the data marked with $*, \dagger$ are not significantly different from each other $(\mathrm{P}>0.05)$.

\subsection{The axial stiffness of the cervical spine}

Under various working conditions, the stiffness of group D was $20 \%$ higher than that of group C and $40 \%$ higher than that of group B. That of group C was $20 \%$ higher than that of group B under fixation. Groups $\mathrm{D}$ and $\mathrm{C}$ had better results than did group $\mathrm{A}$, the blank control group. There were statistically significant differences $(\mathrm{P}<0.05)$ (Table 2).

Table 2 Axial stiffness results of the C5-C7 segment during neck thrust under $150 \mathrm{~N}$ load (x (- $\pm \mathrm{s}, \mathrm{n}=6, \mathrm{~N} / \mathrm{mm})$

\begin{tabular}{|c|c|c|c|c|}
\hline Exercise type & Group A & Group B & Group C & Group D \\
\hline A.C & $35.17 \pm 3.08 * \dagger$ & $39.36 \pm 2.09^{*} \dagger$ & $56.35 \pm 2.93 *$ & $82.75 \pm 3.84 *$ \\
\hline Flex & $22.60 \pm 2.60 *$ & $26.25 \pm 0.98^{*}$ & $32.91 \pm 2.45^{*}$ & $48.50 \pm 2.51^{*}$ \\
\hline Ext & $37.13 \pm 2.12 *, \dagger$ & $34.63 \pm 2.20 *, \dagger$ & $47.58 \pm 4.12 *$ & $62.66 \pm 3.87 *$ \\
\hline LB & $30.48 \pm 1.16^{*}$ & $33.97 \pm 2.37 *$ & $46.32 \pm 4.48^{*}$ & $59.48 \pm 3.17 *$ \\
\hline
\end{tabular}

The relative differences between the data marked with * are statistically significant $(\mathrm{P}<0.05)$, the differences between the data marked with * and those marked with $*, \dagger$ are statistically significant $(\mathrm{P}<0.05)$, and the data marked with $*, \dagger$ are not significantly different from each other $(\mathrm{P}>0.05)$.

\subsection{Torsion mechanical properties of cervical spine}

Under $150 \mathrm{~N}$ of external load and a relative torsion angle $\theta=1.0 \% \mathrm{~cm}$, the torsion value of group $\mathrm{D}$ was $21 \%$ higher than that of group $\mathrm{C}$ and $40 \%$ higher than that of group B, that of group C was $30 \%$ higher than that of group $\mathrm{B}$, and the difference was statistically significant $(\mathrm{P}<0.05)$ (Table 3).

Table 3 Comparison of the torque and torsional stiffness values with various methods internal fixation of the cervical spine $(\mathrm{x}(-) \pm \mathrm{s}, \mathrm{n}=6)$

\begin{tabular}{llc}
\hline Group & Torque $(\mathrm{N} / \mathrm{m})$ & Torsional stiffness $(\mathrm{N} * \mathrm{~m} / \mathrm{rad})$ \\
\hline A & $2.14 \pm 0.15^{*, \dagger}$ & $19.11 \pm 0.71^{*, \dagger}$ \\
B & $2.30 \pm 0.15^{*, \dagger}$ & $19.38 \pm 1.00^{*, \dagger}$ \\
C & $3.42 \pm 0.23^{*}$ & $27.34 \pm 1.12^{*}$ \\
D & $4.35 \pm 0.20^{*}$ & $35.79 \pm 1.66^{*}$ \\
\hline
\end{tabular}

The relative differences between the data marked with * are statistically significant $(\mathrm{P}<0.05)$, the differences between the data marked with $*$ and those marked with $*, \dagger$ are statistically significant $(\mathrm{P}<0.05)$, and the data marked with $*, \dagger$ are not significantly different from each other $(\mathrm{P}>0.05)$.

\section{Discussion}

\subsection{Biomechanical advantages of the anterior cervical pedicle screw plate system}

Anterior cervical fusion with internal fixation is an important clinical treatment for anterior 
compression and restores stability [23,24]. The traditional anterior fixation method mostly uses vertebral screws, which can only yield single-cortical fixation and cannot yield satisfactory stability for long-segment fixation and osteoporotic vertebrae [25]. ACPSs yield high levels of stability through the pedicle and high levels of axial pull-out resistance. Koller et al. [16] directly compared the extraction strength of anterior pedicle screws and anterior vertebral body screws and concluded that anterior pedicle screws can yield strong fixation through the pedicle. Moreover, the results of this study suggest that anterior pedicle screws can be used in a new method of anterior cervical fixation in clinical applications [15]. Wang Yuanzheng et al. [14] also proved that ACPSs are feasible for treating C3-C7. ACPSs can be used in the lower cervical spine. Koller et al. [17] compared the biomechanical properties of a C4-C7 ACPS internal fixation model based on a cadaver specimen with five other reconstruction methods (anterior titanium plate internal fixation, posterior lateral mass screw fixation, posterior $\mathrm{C} 5+\mathrm{C} 6$ lateral mass screw and end use unilateral pedicle screw or bilateral pedicle screw fixation, combined anterior and posterior $360^{\circ}$ reconstruction). It is believed that in addition to anterior titanium plate internal fixation, other cervical spine reconstruction methods can significantly reduce the range of intervertebral motion compared with the initial state. The above mentioned in vitro biomechanical experiments showed that the two internal fixation models of combined anterior and posterior $360^{\circ}$ reconstruction and ACPS reconstruction have obvious differences in flexion and extension but no significant differences in rotation [18-20]. In addition, compared with traditional anterior fixation methods, the stability of ACPS reconstruction in three-dimensional space is significantly better than that of pure anterior titanium plate internal fixation [21].

However, for cervical spine surgery after multisegment subtotal vertebral body resection and total cone resection, ACPS fixation alone and anterior and posterior $360^{\circ}$ reconstruction of the cervical spine also yield good biomechanical stability. This anterior cervical fixation technique should be used more commonly in clinical practice. However, a corresponding transpedicular fixation nail plate system suitable for the anterior cervical spine is clinically lacking.

In this study, a new type of anterior transpedicular fixation nail plate system was developed based on the cervical spine anatomy studied previously [26]. Moreover, 4 pedicle screws or 2 pedicle screws +4 vertebral screws were used. The experimental results indicate that both fixation methods can significantly improve the stability of the injured cervical spine in all directions, but 2 pedicle screws +4 vertebral screws was the most advantageous method. According to the results, the fixation method with 2 pedicle screws +4 vertebral screws has obvious advantages in the number of fixation screws. Second, the combination of vertebral screws and pedicle screws can yield cross fixation, which effectively improves biomechanical stability and resistance. The pull-out force is high, which also effectively improve rotation stability.

\subsection{The surgical indications and biomechanical requirements of cervical internal fixation}

ACPSs are not used in conventional anterior cervical internal fixation. Compared with conventional anterior cervical titanium plate screw internal fixation, fixation with ACPSs has particularities. The specific indications are as follows: (1) the cervical vertebral body is severely damaged by infection, tumour, etc., and cannot be fixed with vertebral screws; (2) anterior cervical multi-stage decompression and bone graft fusion or conventional titanium plate vertebral screws cannot be used due to the state of the patient; (3) single-segment or multi-segment cervical fractures or dislocations that require anterior cervical reduction and internal fixation are present; (4) severe cervical osteoporosis is present; (5) ankylosing spondylitis or conditions where anterior osteotomy is 
required are present; and (6) anterior cervical internal fixation requires revision, and the second operation cannot provide high stability.

The biomechanics of spinal internal fixation devices are used to evaluate the state of internal fixation. The most important experiment is the intensity experiment. Ideally, the elastic modulus is similar to that of bone tissue or internal fixation, and good fatigue resistance and mechanical strength are needed to provide long-term stability for bone graft fusion.

\subsection{Evaluation of the new anterior cervical internal fixation system}

The new type of cervical spine anterior titanium plate was designed with elliptical nail holes in the upper areas of both ends, which is convenient for the placement of unilateral pedicle screws in C34 vertebral bodies and can also be implanted with bilateral pedicle screws in C5-7. The vertebral body of a unilateral pedicle screw can be implanted with two vertebral screws to enhance the stability of the fixed segment. At the same time, the angle of the locking sleeve of the nail plate can be customized through preoperative three-dimensional simulation, which is convenient for intraoperative nail placement. Internal fixation with points and stripes contacting the titanium plate can promote blood circulation in the cortical bone under the titanium plate [22], which promotes fracture healing. In addition, the titanium plate has a curvature in the coronal and sagittal planes to adapt to the physiological curvature of the cervical spine, which can effectively prevent problems such as uneven load and stress distributions, thereby reducing the risk of complications such as screw loosening.

The specimens used in this study were normal cervical spine specimens, so ideally, the role of anterior internal fixation with titanium plates in the restoration of cervical spine stability is reflected in the results. The experimental results suggest that the three fixation methods studied can significantly improve stability in all directions in individuals with cervical spine injuries, but the effect of the method used in group D was the largest.

Anterior pedicle screws can be placed with navigation systems and other auxiliary systems [28-29]. In the early stage of this project, a new method of placing anterior pedicle screws was developed with 3D printing technology [27] and studied. To achieve satisfactory results, the nail plate thread designed in this study was used to help lock the guide sleeve. The direction of the locking sleeve was determined according to the three-dimensional CT images of the specimen before the operation to improve the accuracy of nail placement.

The experimental results show that the new anterior cervical pedicle internal fixation system can provide sufficient stability for simple cervical anterior column instability and has the best antiextension effect. It stabilizes the cervical spine well and has sufficient pull-out strength and fatigue strength. In this study, the appropriate nail plate length and nail hole position were measured according to the three-dimensional data of the specimen, and metal 3D printing was used to make the nail plate.

This experiment proves that the new cervical spine anterior fixation method with a titanium plate through the pedicle can more effectively restore the stability of the cervical spine, provide better stability than traditional anterior cervical nail plate fixation can, and be used to design the locking sleeve. It can effectively improve the accuracy of nail placement and serve as a reliable internal fixation system for anterior cervical pedicle fixation in clinical practice.

\section{References}

[1] Einsiedel T' Schmelz A, Arand M, et al. Injuries of the cervical spine in patients with 
24 ankylosing spondylitis: experience at two trauma centers. Neurosurg Spine, 2006, 5: 33-45.

[2] Koller H, Acosta F, Hempfing A, et al. Cervical anterior transpedicular screw Fixation. Part I: Study on morphological feasibility, indications, and technical prerequisites. Eur Spine J, 2008, 17: 523-538.

[3] Ding Ning, Yuan Feng, et al. Application research of anterior cervical reverse pedicle fixation technique [D]. Xuzhou: Xuzhou Medical College, 2011.

[4] Akula M, Taha M, Mathew B, et al. The Plate cage Benezech implant as an alternative to autologous bone graft in the treatment of cervical spondylosis: clinical and functional outcome. Br J Neurosurg , 2008, 22(4): 542-45.

[5] Arts MP, Brand K Van den Akker E, et al. NE therlands Cervical Kinematics(NECK) Trial. Cost-effectiveness of anterior cervical discectomy with or without interbody fusion and arthroplasty in the treatment of cervical disc herniation; a double-blind randomised multicenter study. BMC Museuloskelet Disord, 2010, 16(11): 122.

[6] Aydogan M, Ozturk C, Karatoprak O, et al. The pedicle screw fixation with vertebroplasty augmentation in the surgical treatment of the severe osteoporotic spines. J Spinal Disord Tech, 2009, 22(6): 444-447.

[7] Barnes AH, Eguizabal Jh, Acosta FJ, ct al.Biomechanical pullout strength and stability of the cervical artificial pedicle screw.Spine(Phila Pa 1976), 2009, 34(1): E16-20.

[8] Becker S, Chavanne A, Spitaler R et al. Assessment of different screwaugmentation techniques and screw designs in osteoporotic spines. Eur SpineJ , 2008, 17(11): 14621469.

[9] Belkoff SM, Mathis JM, Erbe EM, et al. Biomechanical evaluation of a new bone cement for use in vertebroplasty. Spine, 2000, 25(9): 1061-1064.

[10] Koller H, Hempfinng A, Acosta F, et al. Cervical anterior transpedicular screw fixation. Part I: Study on morphological feasibility, indications and technical prerequisites. EurSpine J, 2008, 17(4): 523-538.

[11] Zhao Liujun, Xu Rongming, Xia Huajie, et al. Comparative study on two methods of lower cervical spine transarticular screw implantation[J]. China Orthopedics and Traumatology, 2009, 22(8): 590-592.

[12] Zhu Qingan, Hu Qingmao, Zhong Shizhen, et al. Spinal three-dimensional motion analysis system and its application in lumbar stability analysis [J]. Chinese Journal of Spine and Spinal Cord, 1995, 5 (4): 253-156.

[13] Vaecaro AR, Balderston RA. Anterior Plate in strimuentation for disorders of the subaxial cervical spine. Clinical Orhtopedics and Related Research . 1997, $325: 112$ 121 .

[14] Wang Yuanzhi, Liu Yang, Deng Zhongliang, et al. Experimental study on anterior pedicle screw placement in the lower cervical spine[J]. Journal of the Third Military Medical University, 2012, 34(18): 1839-1843.

[15] Brazenor GA. Comparison of multisegment anterior cervical fixationusing bone strut graft versus atitanium rod and buttress prosthesis: analysis ofoutcome with long term follow-up and interview by independent physician. Spine, 2007, 32(1): 63-71.

[16] KollerH, Acosta F, TauberM , et al. Cervical anterior transpedicular screw fixation 
(ATPS)-Part II , Accuracy of manual insenion and pull-out strength of ATPs. Eurspine J, 2008, 17(4): 539-555.

[17] Koller H, Schmidt R, Mayer M, et al. The stabilizing potential of anteriorposterior and combined techniques for the reconstraction of a 2-level cervical corpectomy model: biomechanical study and first results of ATPS prototyping. Eur Spine J, 2010, 19(12): 2137-2148.

[18] Shahrokni M, zhu Q, Liu J, et al. Design and biomechanical evaluation of a rodent spinal 6xation device. J Spinal Cord, 2012, 50(7): 543-547.

[19] Wang CS, Liu MJ, Lin JH, et al. One stage anterior-posterior approach for traumatic atlantoaxial instability combined with subaxial cervical spinal cordinjury. Chin J Traumatol, 2011, 14(3): 137-142.

[20] Hussain M, Nassr A, Natarajan RN, et al. Biomechanical effects of anterior, posterior, and combined anterior-posterior instrumentation techniques on the stability of amultilevel cervical corpectomy construct: a finite element model analysis. Spine J, 2011, 11(4): 324-330.

[21] Onibokun A, Khoo LT, Bistazzoni S, et al. Anatomical considerations for cervical pediclescrew insertion the use of multiplanar computerized tomography measurements in 122 consecutive clinical cases. Spine J, 2009, 9(9): 729-734.

[22] Wu Xuehui, Li Qihong, Yang Liu, et al. Experimental study on the effect of conical point contact plate internal fixation on local cortical bone microcirculation[J]. Chinese Journal of Orthopaedics, 2000, 20(2): 237-141.

[23] He S, Feng H, Lan Z, et al. A Randomized Trial Comparing Clinical Outcomes Between Zero-Profile and Traditional Multi-Level Anterior Cervical Discectomy and Fusion Surgery for Cervical Myelopathy. Spine, 2018, 43(5): E259- E266.

[24] Donnarumma P, Bozzini V, Rizzi G, et al. Surgical management of C-type subaxial cervical fractures using eervical traction followed by anterior cervical diseeetomy and fusion within $12 \mathrm{~h}$ after the trauma. J Cranio Vertebral Junction Spine, 2017, 8(4): 338-341.

[25] McClelland S 3rd, Passias PG, Errico TJ, et al. Inpatient versus Outpatient Anterior Cervical Discectomy and Fusion: A Perioperative Complication Analysis of 259, 414 Patients From the Heahhcare Cost and Utilization Project Databases .Int J Spine Surg, 2017, 11: 11.

[26] Deng Bin, Yuan Feng, Guo Kaijin, et al. Anatomical study of anterior cervical reverse pedicle screw internal fixation in the lower cervical spine[J]. Journal of Xuzhou Medical College, 2010, 30(8): 520-523.

[27] Sheng Xiaolei, Yuan Feng, Li Zhiduo, et al. Accuracy comparison of 3D printing combined guide plate assisted lower cervical anterior pedicle screw placement and manual placement[J]. Chinese Tissue Engineering Research, 2017, 21(3) : 406-411.

[28] Patton AG, Morris RP, Kuo YF, et al. Accuracy of fluoroscopy versus computerassisted navigation for the placement of anterior cervical pedicle screws[J].Spine (Phila Pa 1976), 2015, 40(7): E404-F410.

[29] Wang Liran, Zhao Liujun, Gu Yongjie, et al. The feasibility of 3D printing navigation template to guide bilateral lower cervical spine anterior pedicle screw placement[J]. Chinese Journal of Spine and Spinal Cord, 2016, 26(11): 1012-1017. 


\section{Declarations}

Ethics approval and consent to participate: Not applicable.

Consent for publication: Not applicable

Availability of data and material: The authors declare that the data and material are true and effective.

Competing Interest: The authors declare that they have no conflict of interest.

Funding: Jiangsu Science and Technology Department Project（ Social developmentClinical Frontier Technology, NO: BE2016647).

Authors' contributions: Maji Sun and Qiuan Wang contributed equally to this work. Maji Sun and Qiuan Wang designed research, performed research, analyzed data, and wrote the paper. Xingchen Zhang and Rui Zhao helped Maji Sun and Qiuan Wang complete the research. Kaijin Guo and Feng Yuan designed research and provided financial support. all authors contributed to the writing and revisions.

Acknowledgements: Thanks to all the authors for their work, and thanks to the Orthopedics and Imaging Department of the Affiliated Hospital of Xuzhou Medical University for their great support. 
Figures
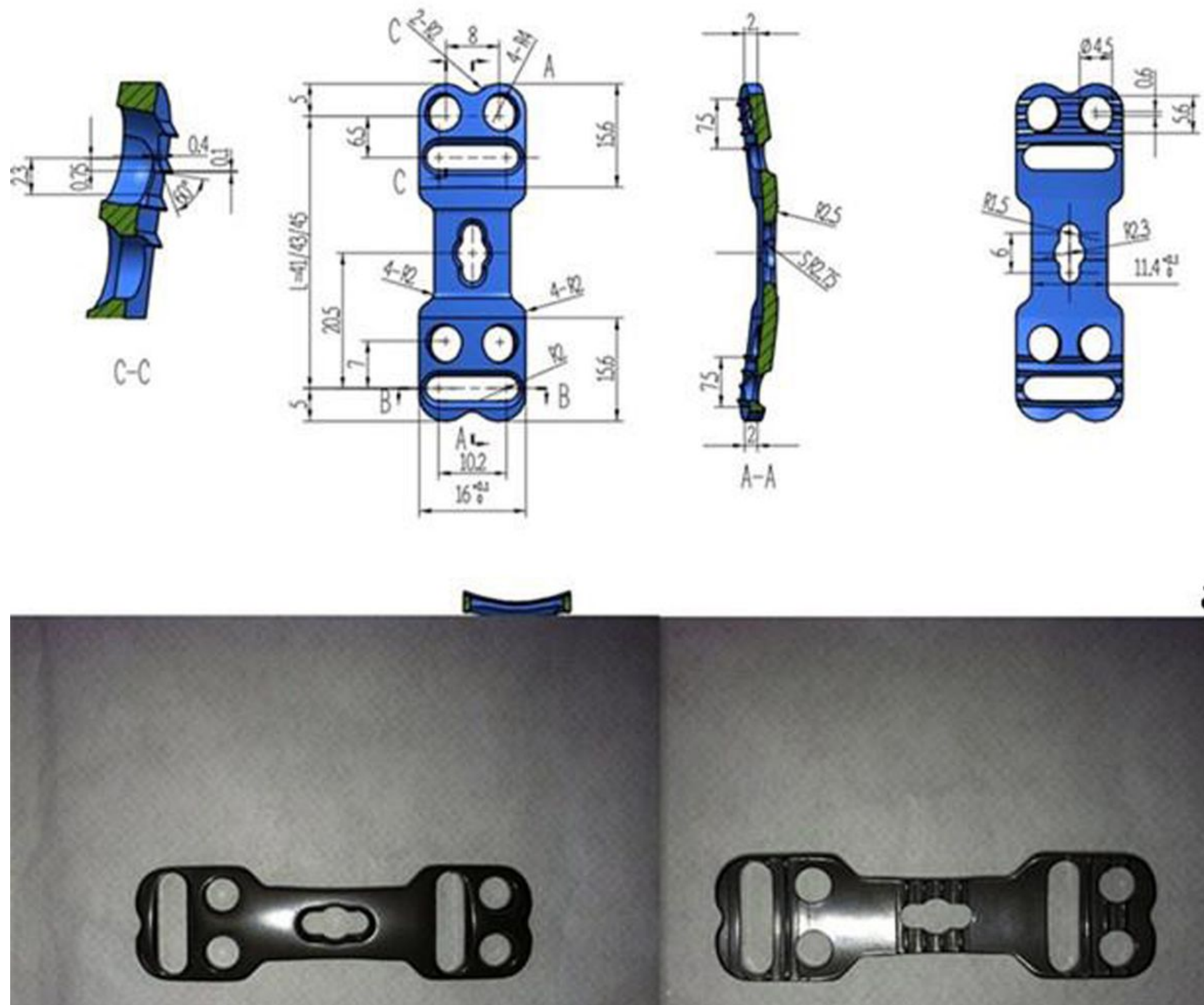

b

\section{Figure 1}

New type of anterior cervical titanium plate. A. Drawing of the titanium plate design B. Front view of the titanium plate $\mathrm{C}$. Back view of the titanium plate 


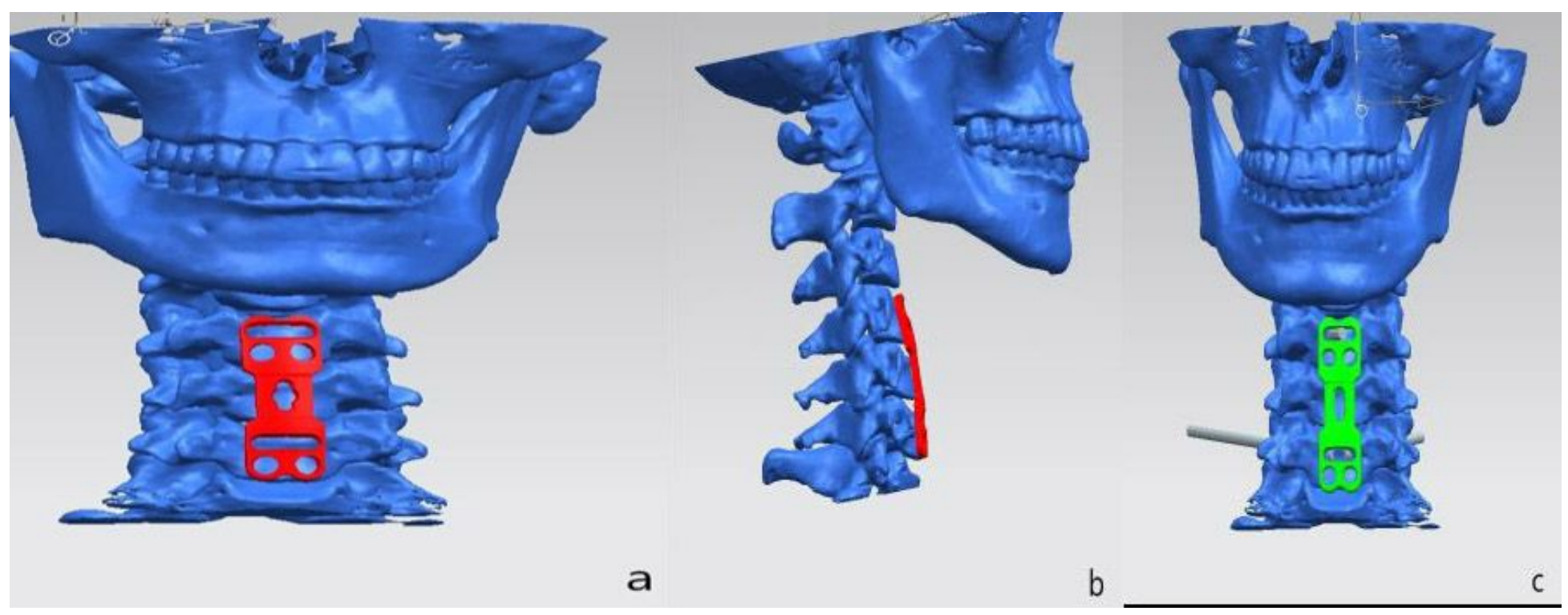

Figure 2

Three-dimensional schematic diagram of the new cervical spine anterior titanium plate fixation method. A Three-dimensional cervical spine simulation showing the placement of the plate; B Three-dimensional cervical simulation showing the lateral placement of the plate; $C$ Three-dimensional simulation of the locking guide sleeve. 


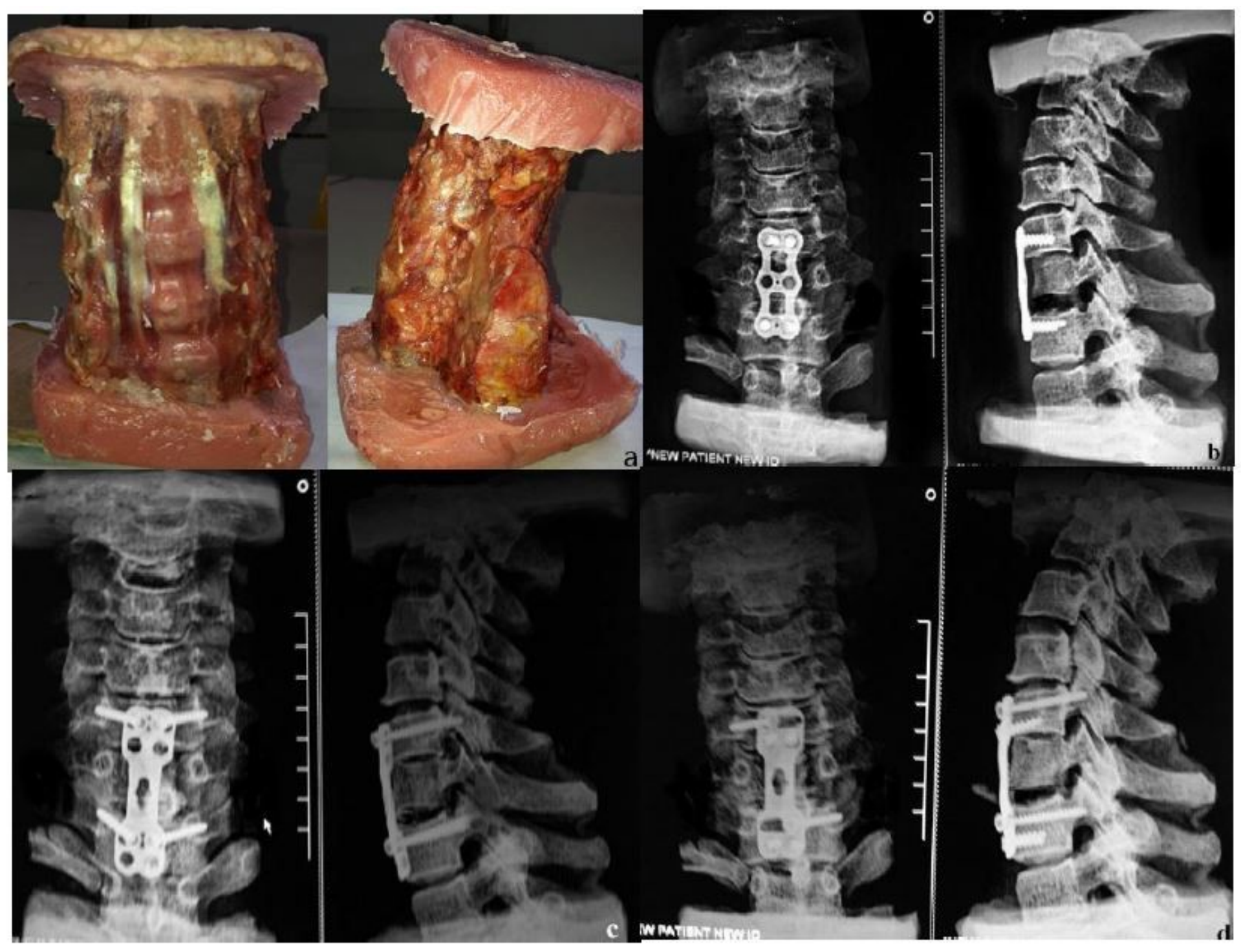

\section{Figure 3}

Postoperative X-ray images of each group in the positive and lateral positions. A. Group A blank control condition; B. Group B fixation method; C. Group C fixation method; D. Group D fixation method. 


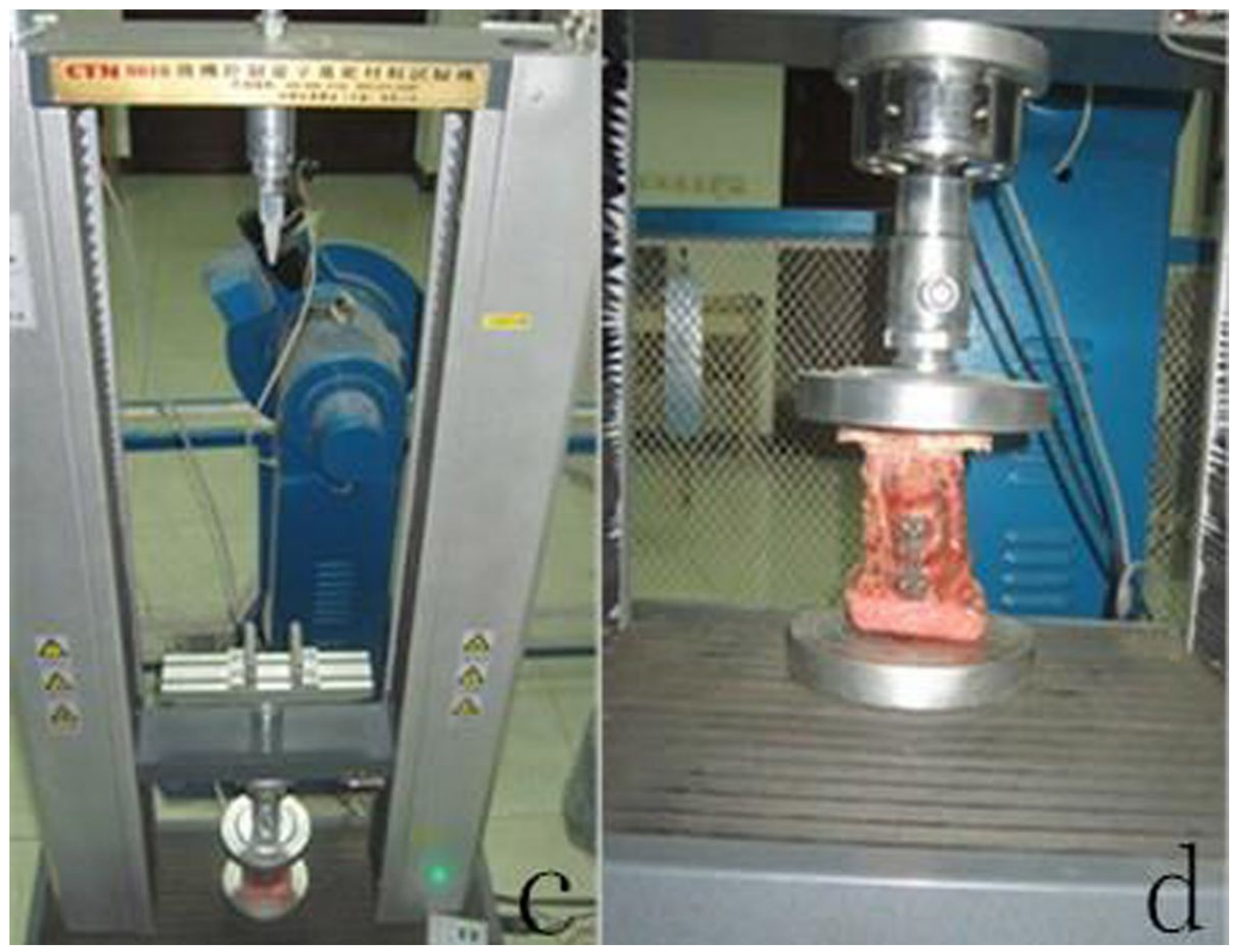

Figure 4

Biomechanical test conducted on a specimen 